\title{
Theoretical and Experimental Investigation on SPR Gas Sensor Based on ZnO/Polypyrrole Interface For Ammonia Sensing Applications
}

\section{AJAY PRATAP SINGH GAHLOT ( $\square$ apsgahlot@db.du.ac.in )}

Deshbandhu College University of Delhi https://orcid.org/0000-0002-9662-6969

\section{Ayushi Paliwal}

Deshbandhu College

Avinashi Kapoor

Department of Electronic Science University of Delhi

\section{Research Article}

Keywords: Gas sensor, Surface Plasmon Resonance, ZnO/polypyrrole composite

Posted Date: November 17th, 2021

DOI: https://doi.org/10.21203/rs.3.rs-1027342/v1

License: (c) (1) This work is licensed under a Creative Commons Attribution 4.0 International License.

Read Full License 


\title{
Theoretical and experimental investigation on SPR gas sensor based on ZnO/Polypyrrole interface for ammonia sensing applications
}

\author{
Ajay Pratap Singh Gahlot ${ }^{1,2^{*}}$,Ayushi Paliwal ${ }^{1}$,Avinashi Kapoor ${ }^{2}$ \\ ${ }^{1}$ Department of Physics, Deshbandhu College, University of Delhi \\ ${ }^{2}$ Department of Electronic Science, University of Delhi. \\ *Email ld-apsgahlot@db.du.ac.in
}

Keywords: Gas sensor; Surface Plasmon Resonance; ZnO/polypyrrole composite.

\begin{abstract}
This work includes the exploitation of laboratory-assembled SPR technique for the application of gas sensor at room temperature. The refractive index change at the interface of $\mathrm{ZnO}$ /Polypyrrole with adsorption of gases $\left(\mathrm{NO}_{2}\right.$ and $\left.\mathrm{NH}_{3}\right)$ is the basis of SPR gas sensor. The theoretical simulations were done to find out the optimum thickness of $\mathrm{ZnO}$ and Polypyrrole composite films for sharp SPR reflectance values. Theoretical SPR curves obtained by changing the value of thickness of Gold nanoparticles film and incident wavelength are also presented in the manuscript. Experimental studies were done to validate the theoretical studies and discussion were done about the interaction of $\mathrm{NH}_{3}$ gas with prism/Au/ZnO/Polypyrrole system. Here, $\mathrm{ZnO} /$ Polypyrrole multilayer structure is the sensing layer to develop highly efficient SPR based $\mathrm{NH}_{3}$ gas sensor. The outcome of these results validate the significance of SPR technique for application of interaction of surface adsorbed analytes, with the interface of dielectrics and sensing material.
\end{abstract}




\section{Introduction}

Research community has always been putting efforts to develop highly sensitive and efficient gas sensors in order to keep the environment and work places safe from any accidental leakage at domestic and Industrial production.[1,2]. At present, we have various technologies of gas sensing which include conductometric and optical sensing technologies. Out of which, conductometric sensing technology has various disadvantages like requirement of elevated temperature demanding high power consumption and poor selectivity. Hence, we need to think for the preference of highly efficient and selective gas sensor based on optical sensing technologies [3]. The major advantage of optical gas sensor is due to its ability of operation, which use less power, at room temperature [4]. A lot of optical techniques are available for sensing purposes such as, FTIR [5], UV-Vis photo spectrometry [6], ellipsometry [7] and Surface Plasmon Resonance technique [8]. Out of all the known techniques, optical gas sensors based on SPR have enormous advantages like simple sample preparation, operation at room temperature, lower detection limit, high selectivity and response. The Surface Plasmon Resonance (SPR) technique is one of the oldest techniques for the application in gas sensing and researchers have exploited it for gas sensing applications $[9,10,11]$. But, results were lacking in terms of poor response and complicated and bulky setup. Therefore, the first step to develop a gas sensor for optimization of thickness of sensing layer. The present work is initiated with theoretical simulation for optimization of thickness of $\mathrm{ZnO}$ layer and Polypyrrole layer separately. SPR technique is very sensitive technique which can be used for sensing of minute changes near the boundary interface of dielectric with the metal [12]. Thus, the variance in the reflectance values can be related for the indication of a particular gas in the vicinity of the interface showing the change of the refractive index.

Now, the focus in this work presented here is on the detection of highly toxic and dangerous ammonia $\left(\mathrm{NH}_{3}\right)$ gas. $\mathrm{NH}_{3}$ gas is produced from as a waste product from livestock or emanation from industry and automobile. Many efforts are continuing in the aim of detection of $\mathrm{NH}_{3}$ gas by research community $[13,14]$. The present focus of the researchers is to develop an efficient gas sensor to sense very low concentration of $\mathrm{NH}_{3}$ leaks $(\leq 5 \mathrm{ppm})$ [15]. There are very few reports available on SPR gas sensors focused for the detection of $\mathrm{NH}_{3}$ gas $[16,17]$. But, they all reported poor sensitivity and aim to detect higher concentration of ammonia ( $>25 \mathrm{ppm})$. Maciak et al. have utilised polyaniline sensing layer based SPR spectroscopy technique to monitor ammonia vapours for a lower concentration value of $32 \mathrm{ppm}$ [16]. Dolbec et al. have also detected higher concentration of ammonia using a different sensing layer i.e. $\mathrm{Nafion} / \mathrm{WO}_{3}$ [17].

This has been previously discussed that gas sensing using SPR technique requires an optimized sensing layer for detection. Hence, choosing a suitable material and its optimization is highly crucial for the development of the sensor. Therefore variation of refractive index in the sensing layer after the exposure of target gas on it, is in direct relation with the detection sensitivity of the analytes interacting with it. For the past few decades, semiconductor metal oxides possess suitable properties for sensing applications i.e. for sensing various toxic gases and among all the explored metal oxides in the application as the sensing material, $\mathrm{ZnO}$ is the best choice. $\mathrm{ZnO}$ has suitable semiconducting, piezoelectric and pyroelectric properties [18]. Another class of materials which recently has been explored are the conducting polymers due to good electrical and optical properties [19]. Out of all the conducting polymers, Polypyrrole (PPy) is the most famous due to stable nature at elevated temperature and easy sample preparation [20]. PPy thin films were prepared electrochemically for $\mathrm{NH}_{3}$ sensing in the concentrations of $0.01-$ $1 \%$ [21]. Yadav et al. have reported $4-8 \%$ of sensing response towards $\mathrm{NH}_{3}$ gas using chemically synthesized Ppy films [22]. Hence, mostly results show low sensing response at 
higher temperature. There is no study available in literature on the development of SPR sensor using $\mathrm{ZnO} / \mathrm{PPy}$ multilayer structure for $\mathrm{NH}_{3}$ gas sensing. This may pave the way of the fabrications of an SPR gas sensor for low level detection of ammonia gas having high sensitivity and efficiency.

In this work, we have done optimization of the thicknesses of $\mathrm{Au}, \mathrm{ZnO} \& \mathrm{PPy}$ thin films theoretically and experimentally for sharp SPR curve. The change in refractive indexes of the $\mathrm{ZnO} /$ Polypyrrole composite multilayer thin film structure deposited at their optimized value of thickness on the of gold were examined using SPR technique with varying concentration of toxic gas like $\mathrm{NH}_{3}$ gas.

\section{Theoretical simulation details}

The behaviour of intensity of reflected light with angle of incidence (angular interrogation mode) can be described by Fresnel's equations where light is reflected form different interfaces (metal/dielectric, $\mathrm{prism} / \mathrm{metal}$, dielectric/dielectric interfaces). The theoretical values of reflectance for single layer (prism/metal/air) and double layer (prism/metal/dielectric/air) systems have applied for the determination of the refractive index of gold(metal) and dielectric layer respectively.

\section{One layer system (prism/metal/dielectric (air))}

Let $n_{i}(\mathrm{i}=0,1,3)$ be the values of refractive index for the any one of the medium(prism $\left./ \mathrm{metal} / \mathrm{dielectric}\right)$, and $\theta_{\mathrm{i}}$ is corresponding value of incident angle at the prism/metal/air boundary of these media (figure 1.11), here we will use the subscripts as 0 corresponding to the prism, as 1 corresponding to metal and 3 as for the dielectric (air) media. The Fresnel's reflection coefficient for a p-polarized light passing from $\mathrm{i}^{\text {th }}$ medium to $\mathrm{k}^{\text {th }}$ medium is given as [23]

$$
r_{i k}=\frac{n_{k} \cos \theta_{i}-n_{i} \cos \theta_{k}}{n_{k} \cos \theta_{i}+n_{i} \cos \theta_{k}}
$$

The $\mathrm{z}$-component of the propagation constant in $i^{\text {th }}$ medium is given by [23]

$$
\mathrm{k}_{\mathrm{zi}}=\frac{2 \pi}{\lambda} \mathrm{n}_{\mathrm{i}} \cos \theta_{\mathrm{i}}=\frac{2 \pi \sqrt{\varepsilon_{\mathrm{i}}-\varepsilon_{0} \sin ^{2} \theta}}{\lambda}
$$

Since, dielectric constant,$\varepsilon=(\text { refractive index }, n)^{2}$ and using equations (1) and (2), we have,

$$
r_{\mathrm{ik}}=\left(\frac{k_{\mathrm{zi}}}{\varepsilon_{i}}-\frac{k_{\mathrm{zk}}}{\varepsilon_{k}}\right) /\left(\frac{k_{\mathrm{zi}}}{\varepsilon_{i}}+\frac{k_{\mathrm{zk}}}{\varepsilon_{k}}\right)
$$

where ' $\varepsilon_{i}$ ' and ' $\varepsilon_{k}$ ' are the dielectric constants of the $i^{t h}$ and $k^{\text {th }}$ medium, and ' $\varepsilon_{0}$ ' is the dielectric constant for the prism.

So, the Reflectance for single layer system (prism/metal/dielectric (air)) is given by [23]:

$$
R_{013}=\left|\frac{r_{01} r_{13} \exp \left(2 i k_{z 1} d_{1}\right)}{1+r_{01} r_{13} \exp \left(2 i k_{z 1} d_{1}\right)}\right|^{2}
$$

where, subscripts ' 0 ', ' 1 ' and ' 3 ' refer to prism, metal layer and dielectric(air) media respectively. ' $d_{1}$ ' is the thickness of metal thin film. ' $r i k$ ' and ' $k_{z i}$ ' are Fresnel's reflection coefficients and $\mathrm{z}$ components of propagation constant as given by equations (3) and (2) respectively. The reflectance is calculated for the single layer system (prism/metal/dielectric (air)) by equation (4) using known values for $\lambda, \theta$, and $\varepsilon$, and value of complex dielectric constants of metal layer may be obtained.

\section{Double layer system (prism-Au-dielectric-air)}

For realization of gas (optical)sensors, a suitable sensing layer (dielectric) has to be integrated with metal layer, which should be sensitive to the stimulus under consideration. Also, to probe the property of any unknown material (dielectric), it has to be in the vicinity of metal layer Therefore, study of double layer system consisting of prism/metal/dielectric thin film/dielectric(air) is important. Using the Fresnel's equation for the calculation of the reflectance of this double layer system is as follows [23]:

$$
R_{0123}=\left|\frac{r_{01}+r_{123} \exp \left(2 i k_{z 1} d_{1}\right)}{1+r_{01} r_{123} \exp \left(2 i k_{z 1} d_{1}\right)}\right|^{2}
$$


where

$r_{123}=\frac{r_{12}+r_{23} \exp \left(2 i k_{z 2} d_{2}\right)}{1+r_{12} r_{23} \exp \left(2 i k_{z 2} d_{2}\right)}$

and $r_{i k}$ are the Fresnel's reflection coefficients given by equations (1) and (3). Here, subscript ' 2 ' refers to the deposited $\mathrm{ZnO} /$ Polypyrrole as sensing material and ' ${ }_{2}$ ' is its depth. Considering the dielectric constant of the sensing layer $\left(\varepsilon_{2}\right)$ as the fitting parameter, the SPR reflectance obtained for $\mathrm{prism} / \mathrm{metal} / \mathrm{dielectric/dielectric(air)} \mathrm{system} \mathrm{is} \mathrm{fitted} \mathrm{with} \mathrm{equation} \mathrm{(5)} \mathrm{and} \mathrm{the} \mathrm{complex} \mathrm{dielectric}$ constant of sensing layer is obtained.

\section{Experimental Details: Fabrication of SPR based $\mathrm{NH}_{3}$ gas sensor}

In this work, multilayer structure of the films was prepared. We deposited the gold(Au) thin film of optimized value on the surface of glass prism by thermal evaporation method on the parameters reported in our previous report [24]. After this, $\mathrm{ZnO}$ film is coated on Au coated prism. Sol-gel method was utilised to synthesis $\mathrm{ZnO}$ nanoparticles and then they were put in thin film form by using spin coating technique [25]. Finally, Polypyrrole(PPy) thin films were coated over this structure. PPy nanoparticles were fabricated by the method of chemical polymerization and their films were prepared by spin coater [25].

We use Kretschmann configuration for the excitation of surface plasmon(SP) on the prism/gold interface by using a BK-7 glass prism $\left(\mathrm{n}_{\mathrm{p}}=1.517\right)$ and the optimized thickness of $\mathrm{Au}$ film with the help of a SPR measurement system. For the measurement of the SPR reflectance, He-Ne laser (p-polarised and of wavelength $633 \mathrm{~nm}$ ) was used for the irradiation on the prism/Au/ZnO/PPy/air system and the reflected light (which is a function of incident angle )was monitored. Schematic of our SPR gas (optical)sensor , as shown in figure 1, uses a cylindrical cell for the target gas attached to the prism deposited with the gold(Au) and the target gas sensitive material's coating (prism/Au/ $\mathrm{ZnO} / \mathrm{PPy} /$ air) is placed in contact with this cylindrical gas cell so that the target gas molecules can interact with the deposited gas sensitive material interface ( $\mathrm{ZnO} / \mathrm{PPy})$. A mechanical rotary pump was used for creating vacuum. Static and dynamic modes measurements were done. The Ammonia $\left(\mathrm{NH}_{3}\right)$ target gas of a specific concentration value was inserted into this gas cell with a calibrated needles connected to inlet valve. In the static mode, we insert the ammonia gas inside the gas cylindrical cell for the full angle variation and the SPR reflectance plot was recorded with an optical power meter. The dynamic measurements were done with the system of prism/ $\mathrm{Au} / \mathrm{ZnO} / \mathrm{PPy} / \mathrm{air}$, where we get a minimum value of SPR reflectance $\left(\mathrm{R}_{\min }\right)$. We than record the intensity of the reflected light keeping incident light at a fixed angle (as a function of time) with the help of a CCD camera interfaced to a computer. The change in intensity is measured for the various concentrations of the ammonia gas, when it is introduced into the gas cell and as well as for outgoing of the ammonia gas. We use rotatory pump for the ejection of ammonia gas for the recovery time measurement of our gas sensor system of ( $\mathrm{prism} / \mathrm{Au} / \mathrm{WO}_{3} / \mathrm{air}$ ), Which then filled with the fresh

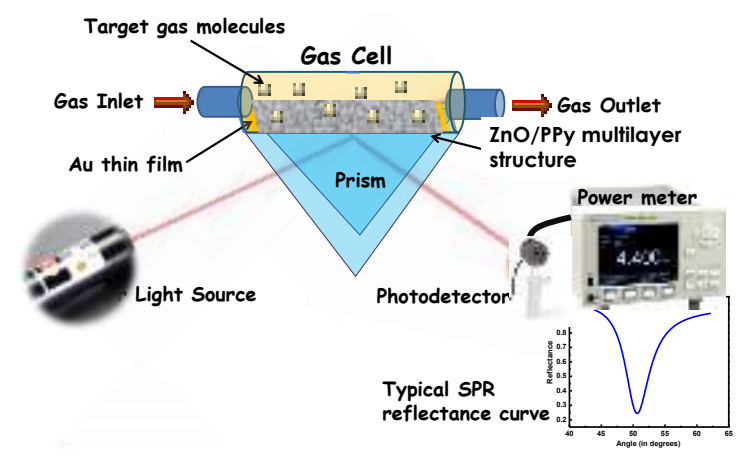

clean air for the reference.

Figure 1: Schematic of the Kretschmann configuration based SPR gas sensor 


\section{Results and discussion}

Theoretical simulation studies

Varying thickness of gold thin film:

SPR reflectance curves by varying the thickness of Au in equation (4) are depicted in figure 2. SPR reflectance sharpest value is observed for $40 \mathrm{~nm}$ thickness of Au having minimum FWHM.
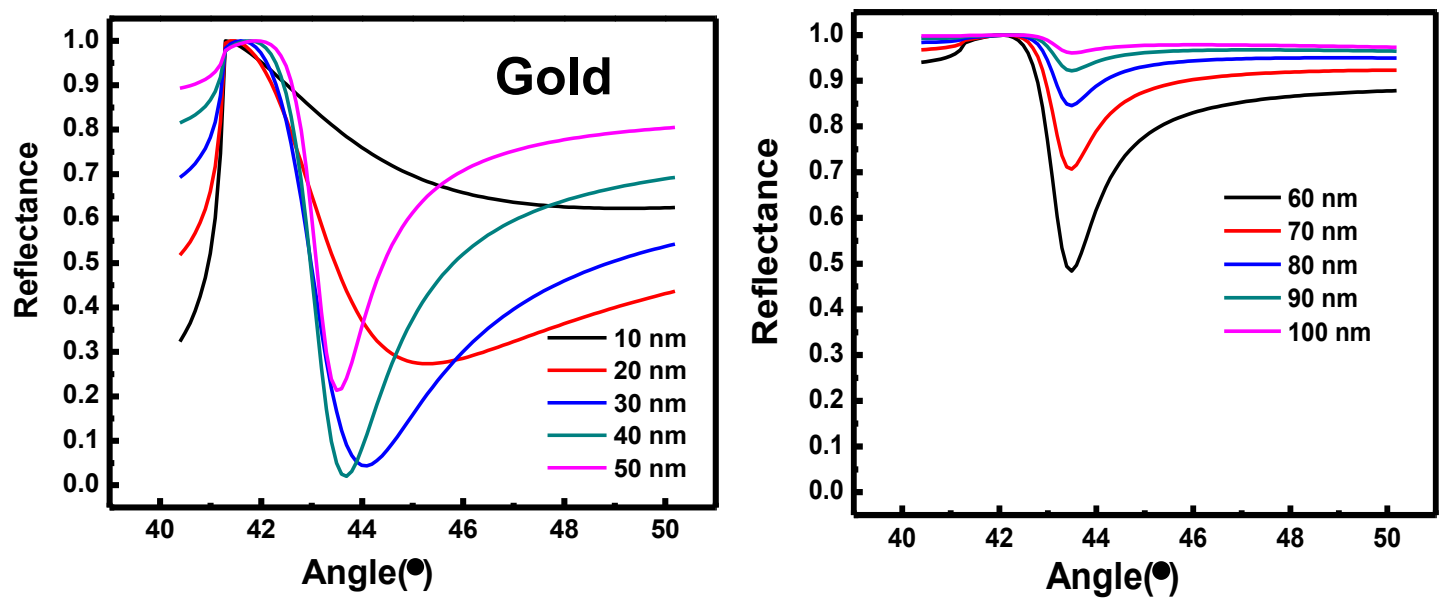

Figure 2: Theoretical SPR reflectance Plots the system of prism/Au/air by changing the thickness of Au layer

SPR reflectance plots of the system prism/Au/air, prism/Au/ZnO/air and prism/Au/ZnO/PPy/air:

Schematic of the two and three layer configuration used is shown in figure 3 (a). Theoretical simulation of the SPR reflectance plots by increasing the number of layers is shown in figure 3(b) (i.e. prism/Au/air, prism/Au/ZnO/air and prism/Au/ZnO/PPy/air). The angle of resonance shifts towards higher values by increasing the number of layers from $\mathrm{ZnO}$ /air interface to $\mathrm{ZnO} / \mathrm{PPy} /$ air interface.

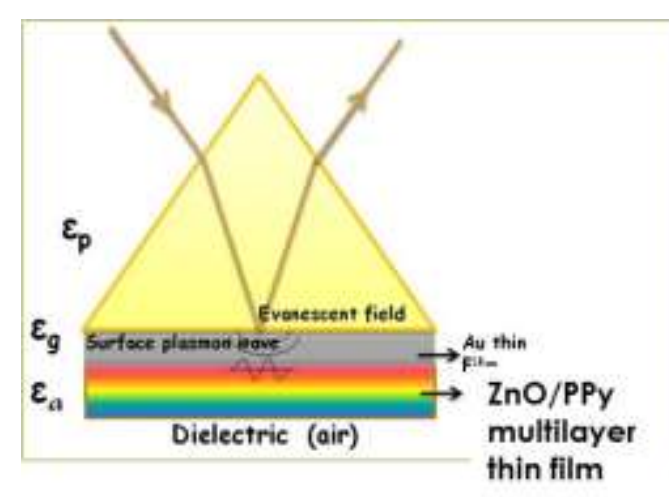

a

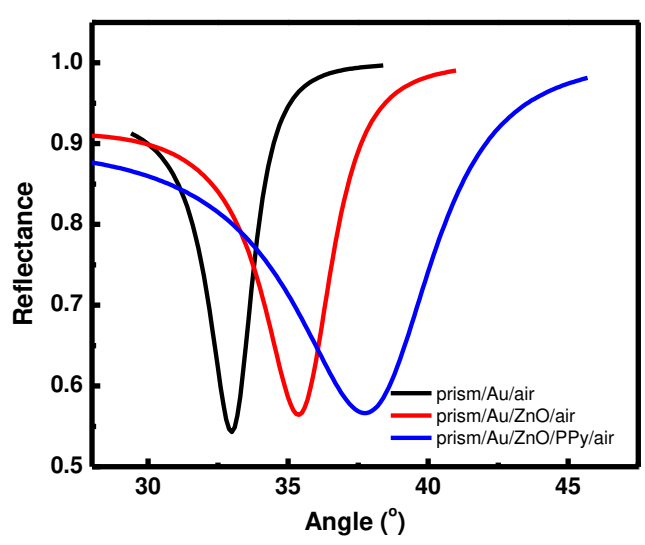

(b) 
Figure 3: (a) Schematic of SPR system used for theoretical simulation, (b) Plots of the SPR reflectance for the system of prism/Au/air, prism/Au/ZnO/air and prism/Au/ZnO/PPy/air

\section{Varying thickness of $\mathrm{ZnO}$ :}

Figure 4 shows the SPR reflectance plots for the system of prism/Au/ZnO/PPy/air by changing the thickness of $\mathrm{ZnO}$.

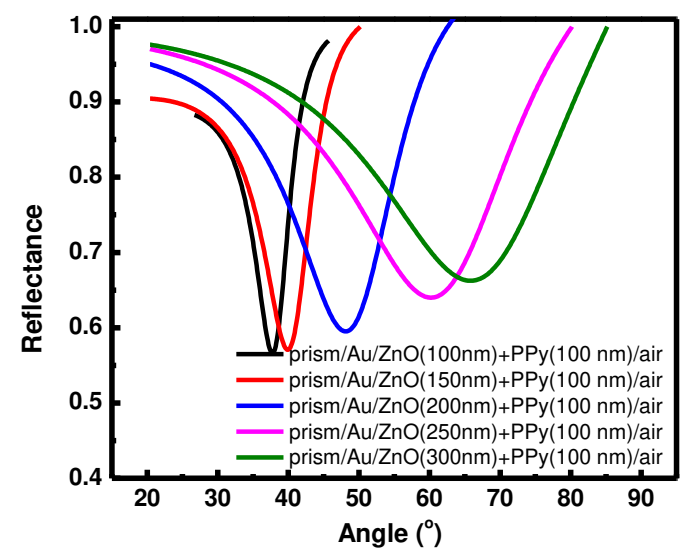

Figure 4: Plots of the SPR reflectance for the system of prism/Au/ZnO/PPy/air by changing the thickness of $\mathrm{ZnO}$

The variation of resonance angle, minimum reflectance and FWHM have been presented in figures 5 (a), (b) and (c) respectively. All the parameters increase with increase in thickness of $\mathrm{ZnO}$. SPR reflectance curve having minimum FWHM is chosen for experimental studies. Hence, $100 \mathrm{~nm} \mathrm{ZnO}$ SPR has minimum FWHM (or absorption losses) is the optimized

thickness of $\mathrm{ZnO}$ layer.
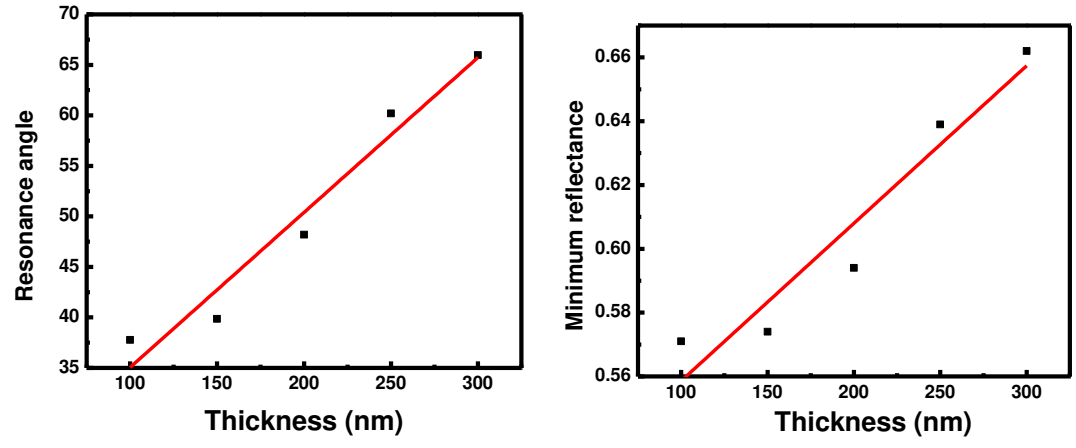

(b)

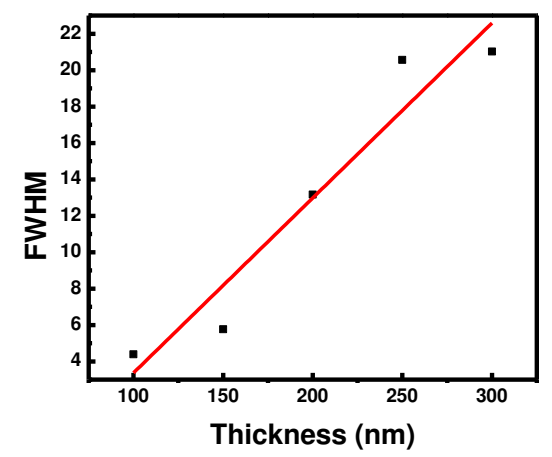

(c) 
Figure 5: Plots of (a) Angle of resonance, (b) minimum reflectance and (c) FWHM with the thickness of $\mathrm{ZnO}$

Varying thickness of Polypyrrole thin film

Figure 6 shows the plots of SPR reflectance of the gas sensing system of prism/Au/ZnO/PPy/air by changing the thickness of PPy.

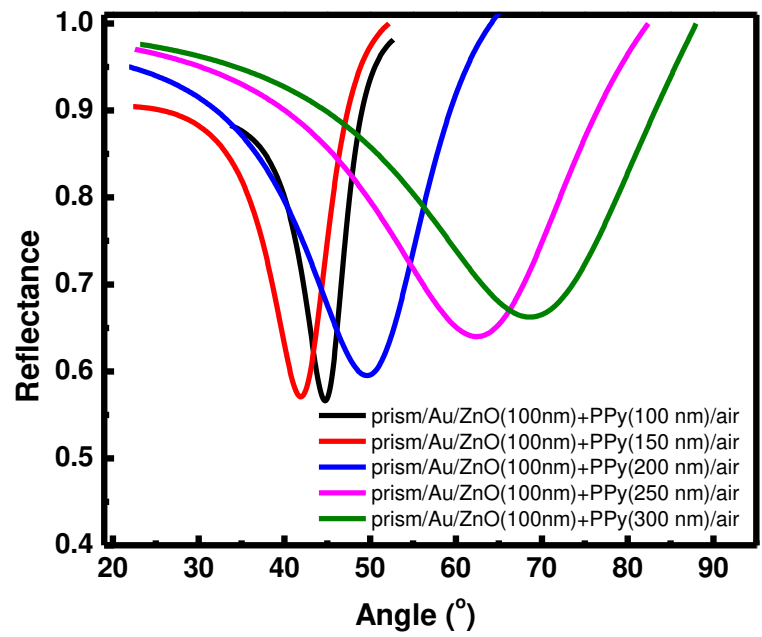

Figure 6: SPR reflectance plots for the system of prism/Au/ZnO/PPy/air by changing the thickness of PPy

The variation of resonance angle, minimum reflectance and FWHM are presented in figures 7 (a), (b) and (c) respectively. All the parameters increase linearly with the increase in thickness of PPy. SPR reflectance curve having minimum FWHM is chosen for experimental studies. Hence, $150 \mathrm{~nm}$ PPy SPR has minimum FWHM (or absorption losses) is the optimized thickness of PPy layer

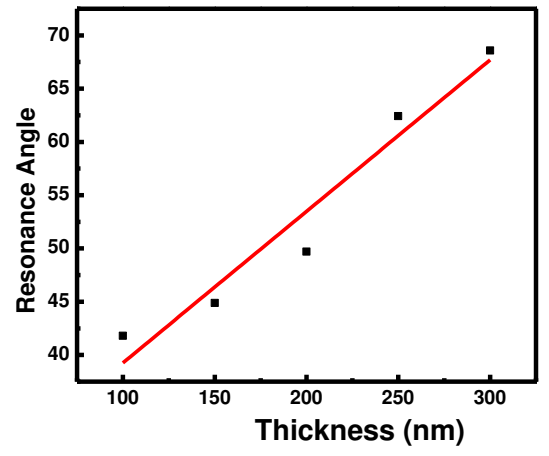

a

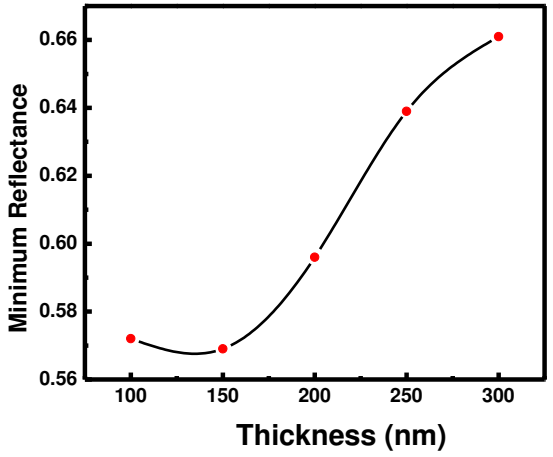

(b) 


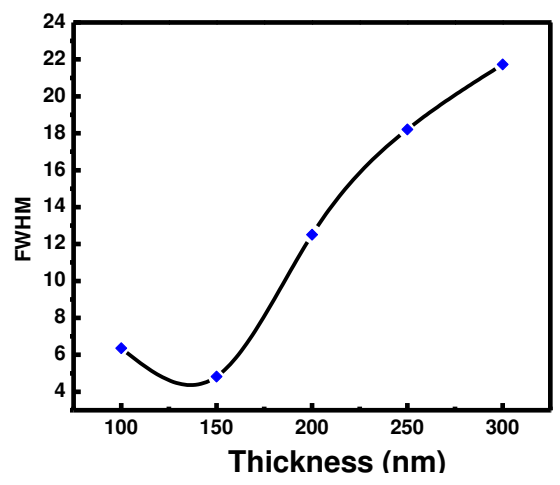

(c)

Figure 7: Variation of (a) resonance angle, (b) minimum reflectance and (c) FWHM with the thickness of $\mathrm{ZnO}$

\section{Varying incident wavelength}

SPR reflectance plots of the optimized system of prism/Au/ZnO/PPy/air with the $100 \mathrm{~nm} \mathrm{ZnO}$ and $150 \mathrm{~nm}$ PPy are shown in figure 8. With increase in the wavelength of the incident light, the resonance angle shifts to lower value. FWHM of the SPR curve for $355 \mathrm{~nm}$ incident wavelength is maximum which decreases with increase in wavelength and is found to be minimum for $633 \mathrm{~nm}$ wavelength. After $633 \mathrm{~nm}$ wavelength, the SPR curves becomes attenuated. The experimentally obtained SPR reflectance curves are shown by dotted lines and theoretically simulated curves by solid line.

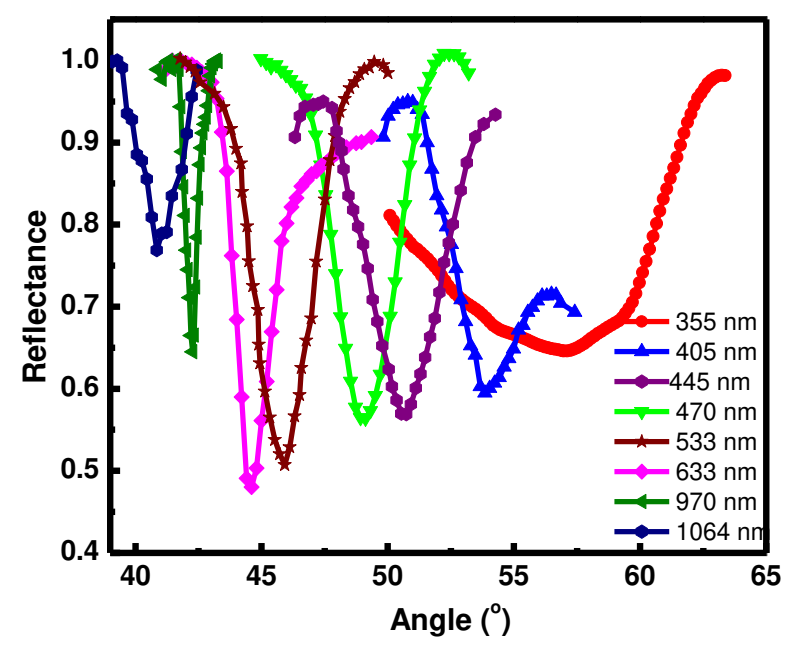

Figure 8: SPR plots for the system of prism/Au/ZnO/PPy/air with different incident

wavelength

Varying effective refractive index

Theoretical simulations were also done on the system of prism/Au/ZnO/PPy/air by varying the refractive index of the exposed sensing material, which is in contact with the $\mathrm{ZnO} / \mathrm{PPy}$ interface on exposure to $\mathrm{NH}_{3}$ gas of different concentration. Refractive index is sequentially 
varied and SPR reflectance curves are measured. It is observed from figure 9 (a) that ATR curves shift towards right i.e., resonance angle increases with increase of the effective refractive index of the sensing material. The linear change of the angle of resonance and minimum reflectance with increase in the effective refractive index is shown in figure 9 (b) and (c) respectively. This confirms the possibility of developing a highly sensitive sensor utilising the system of prism/Au/ZnO/PPy/air as a detector for the Ammonia( $\left.\mathrm{NH}_{3}\right)$ gas with the varying concentration.

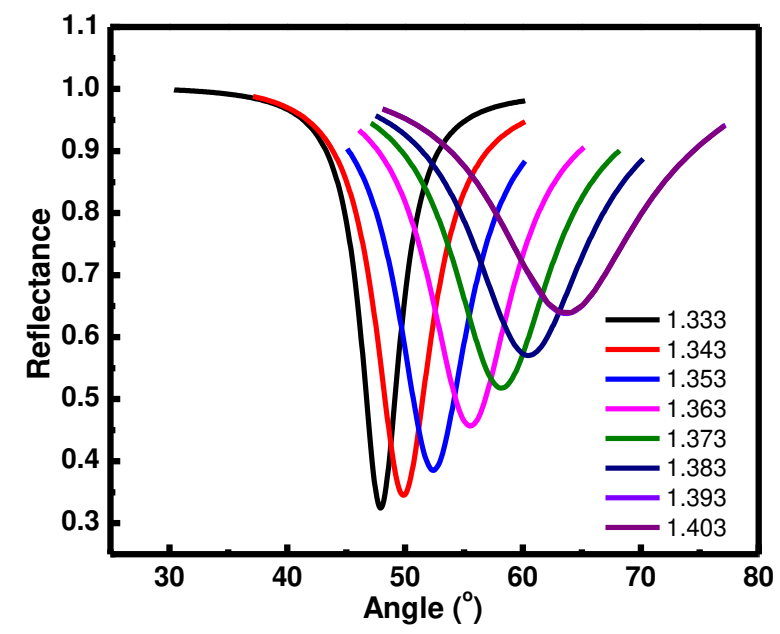

(a)

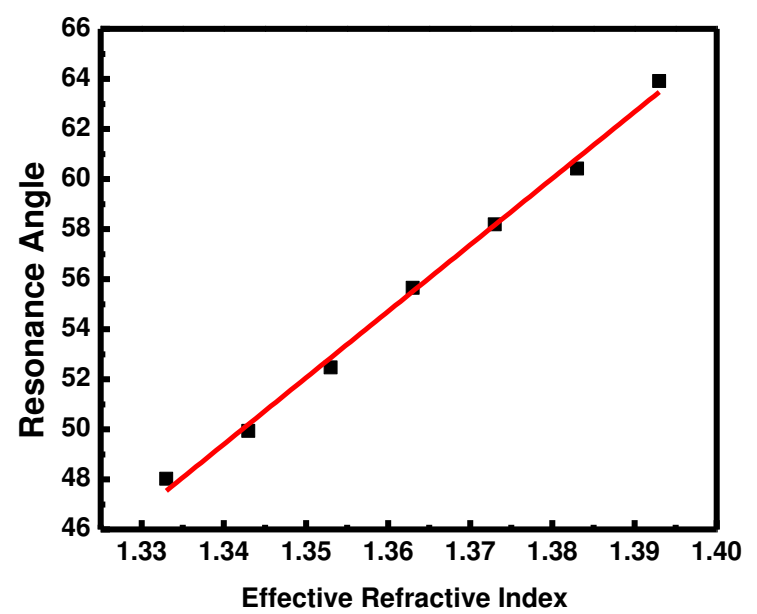

b

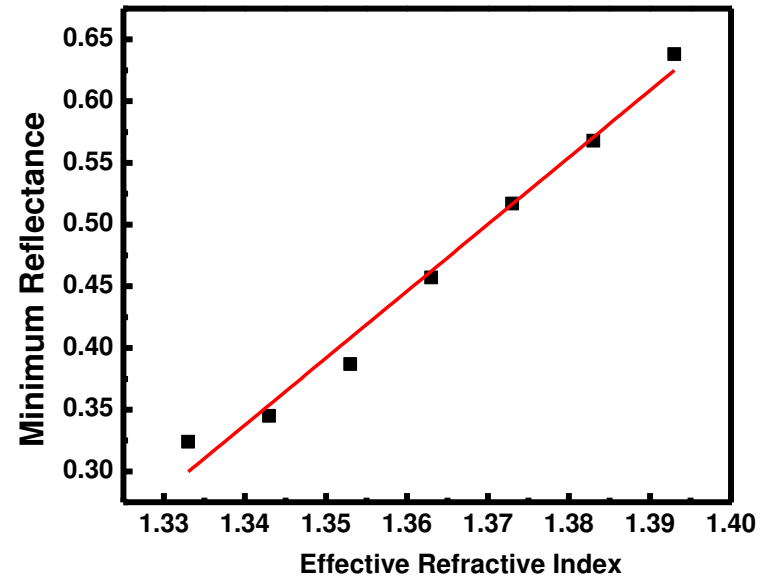

(c)

Figure 9: (a) SPR reflectance plots of the system of prism/Au/ZnO/PPy/air for varying effective refractive index, (b) Linear increase of resonance angle and (c) the minimum reflectance with changing effective refractive index

\section{Experimental studies}


The data of SPR reflectance obtained for the system of prism/ $\mathrm{Au} / \mathrm{ZnO} / \mathrm{PPy} / \mathrm{NH}_{3}$ when exposed to changing concentration of $\mathrm{NH}_{3}$ gas $(0$ to $200 \mathrm{ppm})$ is plotted in figure 10. A continuous change in $\theta_{\mathrm{SPR}}$ towards higher values of angles(from $54.9^{\circ}$ to $95.3^{\circ}$ )is observed with the incremental change of the concentration of $\mathrm{NH}_{3}$ gas from 1 to $200 \mathrm{ppm}$ (figure 10).

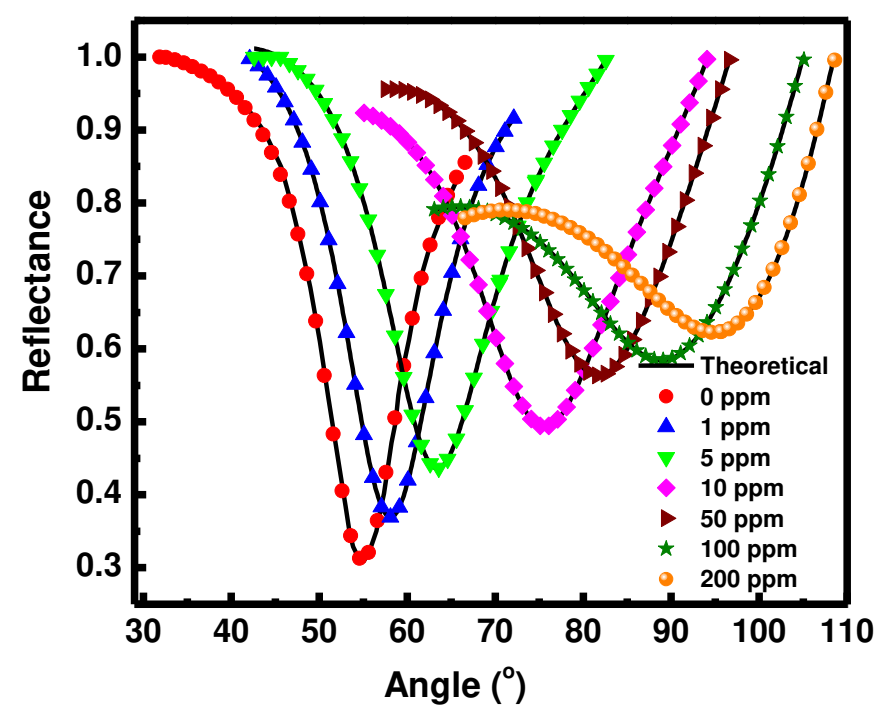

Figure 10: Experimental data obtained for the system of prism/ $\mathrm{Au} / \mathrm{ZnO} / \mathrm{PPy} / \mathrm{NH}_{3} \mathrm{SPR}$ reflectance plots upon exposure of $\mathrm{NH}_{3}$ gas with increasing concentration the theoretical value is also plotted for comparison.

Figure 11 (a) and (b) depict the exponential increment of the values of $\theta_{\mathrm{SPR}}$ and $\mathrm{R}_{\min }$ for our gas sensing system of prism/ $\mathrm{Au} / \mathrm{ZnO} / \mathrm{PPy} / \mathrm{NH}_{3}$ when we vary the concentration of $\mathrm{NH}_{3}$ gas from 0 to $200 \mathrm{ppm}$. The observed change of the SPR data is due to the variation of the effective refractive index of $\mathrm{ZnO} / \mathrm{PPy}$ interface when the ammonia gas is adsorbed at various concentrations. As evident from figure 11 (a) and (b), there is linear increase in $\theta_{\text {SPR }}$ and $R_{\min }$ till 10 ppm concentration of the Ammonia $\left(\mathrm{NH}_{3}\right)$ gas. As the concentration of the Ammonia gas increase, the values of $\theta_{\mathrm{SPR}}$ and $\mathrm{R}_{\min }$ saturates. This is due to the fact that with the high Ammonia gas concentration, there is no appreciable change in effective refractive index which is reflected in the nonlinear variation of $\theta_{S P R}$ and $R_{\min }$. We can also see, for the FWHM of SPR response of sensing system shows the same variation as that of $\theta_{\mathrm{SPR}}$ and $\mathrm{R}_{\min }$ (figure 11 (c)), showing that the losses increase continuously until $10 \mathrm{ppm}$ concentration is reached. 

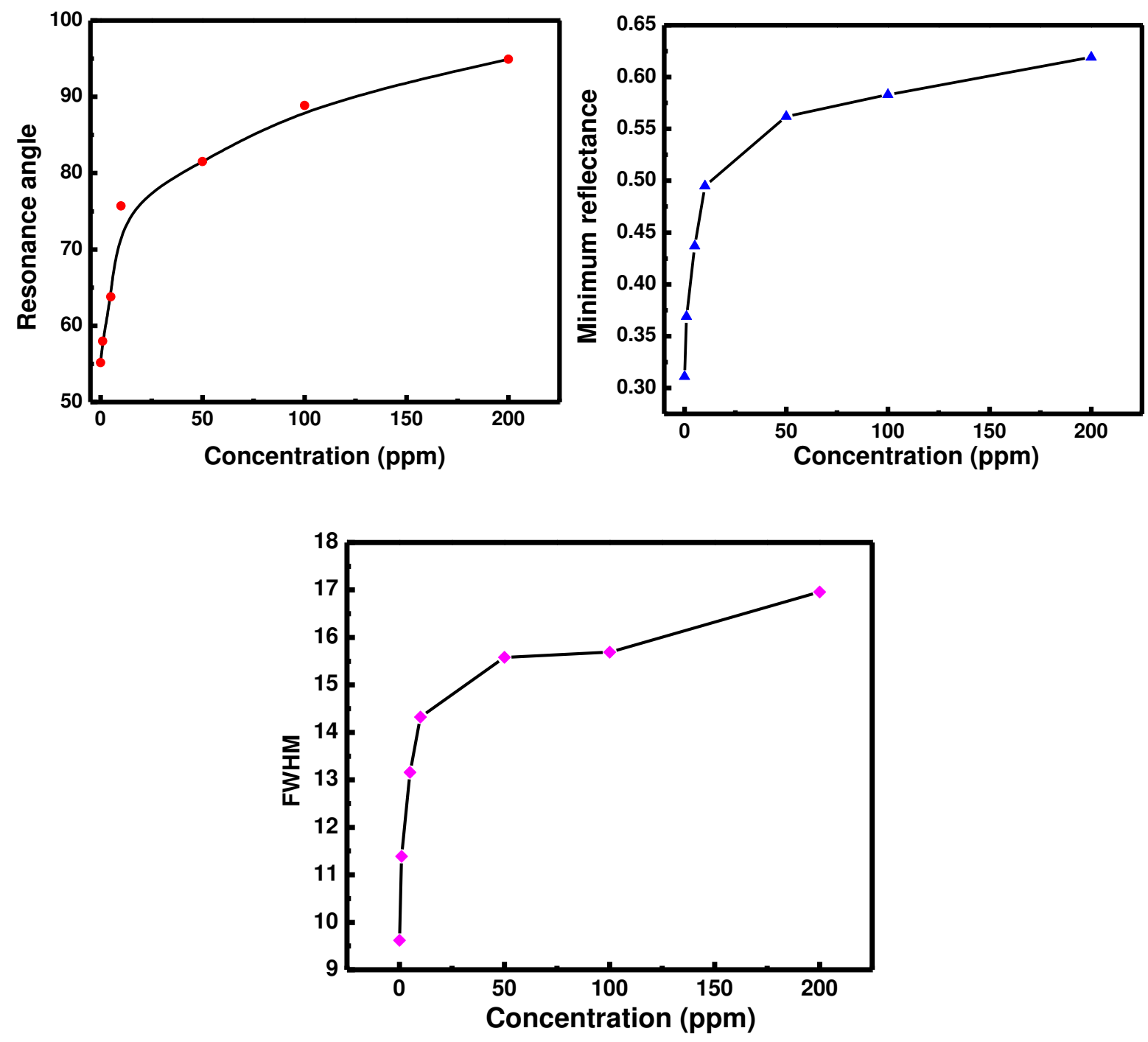

Figure 11: Plots of (a) SPR angle of resonance $\left(\theta_{\mathrm{SPR}}\right)$, (b) $\mathrm{R}_{\min }$, and (c) FWHM of the SPR sensing system with variation in the concentration of $\mathrm{NH}_{3}$ gas

The experimental data for the SPR reflectance measured in the presence of $\mathrm{NH}_{3}$ gas of various concentrations has been fitted theoretically with the help of Fresnel equations as mentioned earlier, and the estimation of the values of $n_{i}$ and $k_{i}$ were done. The variation with the effective index of $\mathrm{ZnO} / \mathrm{PPy}$ interface when the adsorbed $\mathrm{NH}_{3}$ gas with its increasing concentration is as shown in figure 12 (a) (calibration curve). The refractive index was found to increase linearly till $10 \mathrm{ppm}$ concentration as shown in figure 12(b). The graph is fitted linearly and the value of sensitivity is found from the slope of the calibration curve i.e. $3.15 \times 10^{-3} \mathrm{RIU} / \mathrm{ppm}$. 

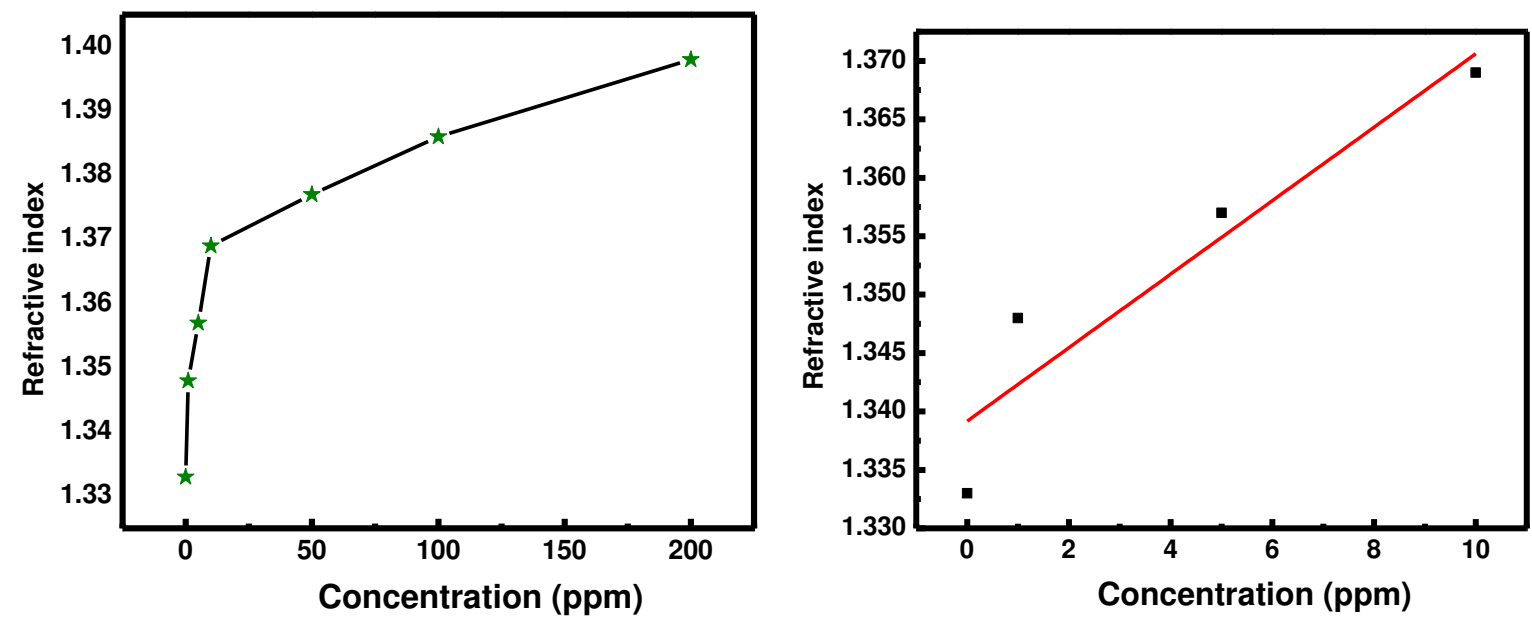

Figure 12: (a) Variation of effective index of $\mathrm{ZnO} / \mathrm{PPy}$ interface when the $\mathrm{NH}_{3}$ gas get adsorbed, with the increase of its concentration and (b) Variation of the refractive index till 10 ppm concentration

When $\mathrm{NH}_{3}$ gas is present in the bulk media, it's adsorption on the $\mathrm{ZnO} / \mathrm{PPy}$ interface leads to the Surface plasmon propagation distortion and so the losses in the gas sensing system keep on increasing as depicted from plots of the increasing FWHM of the SPR reflectance curves. We find the important fact that to the gas sensing system of prism/Au/ZnO/PPy shows good sensitivity and high linearity over a wide good range (1 to $10 \mathrm{ppm}$ ) of $\mathrm{NH}_{3}$ gas.

Figure 13 (a) depicts the dynamic response for the system of prism/Au/ZnO/PPy upon exposure with the changing concentration of $\mathrm{NH}_{3}$ gas from 0 to $200 \mathrm{ppm}$ and the incident angle is kept at value of $\theta=54.9^{\circ}$. Here we use a CCD for measuring the changes in the reflectance of our gas sensing system. The gas sensing system shows a continuous increase in the sensitivity (change in reflectance) with the change in concentration of $\mathrm{NH}_{3}$ gas from 1 to $200 \mathrm{ppm}$ kept at the room temperature (figure 13 (a)). The calibration curve of the SPR gas sensor is also plotted as shown in the figure 13 (b). Figure 13 (b) shows that there is a saturation of change in reflectance after $10 \mathrm{ppm}$ concentration. A linear increase in the response i.e., change in reflectance with increase in concentration of $\mathrm{NH}_{3}$ gas over the range 1 to $10 \mathrm{ppm}$ is shown in figure 13(c). The variation in sensing response $(\Delta R)$ is similar to that obtained for $R_{\min }$ and $\theta_{\text {SPR }}$ with $\mathrm{NH}_{3}$ gas concentration. The sensitivity of the fabricated SPR sensor prism/Au/SnO 2 is about $0.112 / \mathrm{ppm}$ for $\mathrm{NH}_{3}$ concentration varying from $1 \mathrm{ppm}$ to $10 \mathrm{ppm}$ (figure 13 (c)). The obtained result represents the development of a sensitive SPR gas sensor for efficient detection of $\mathrm{NH}_{3}$ gas. 


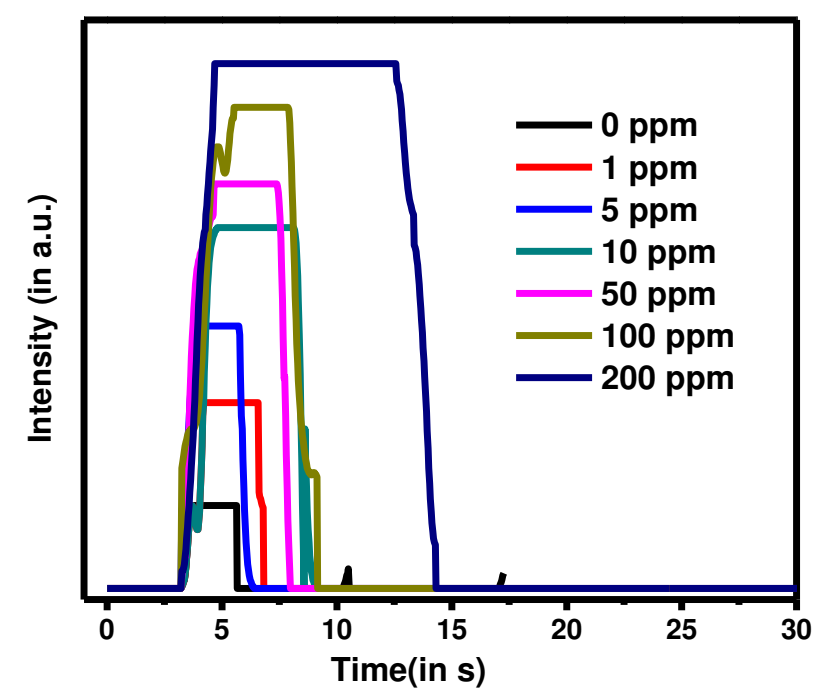

(a)

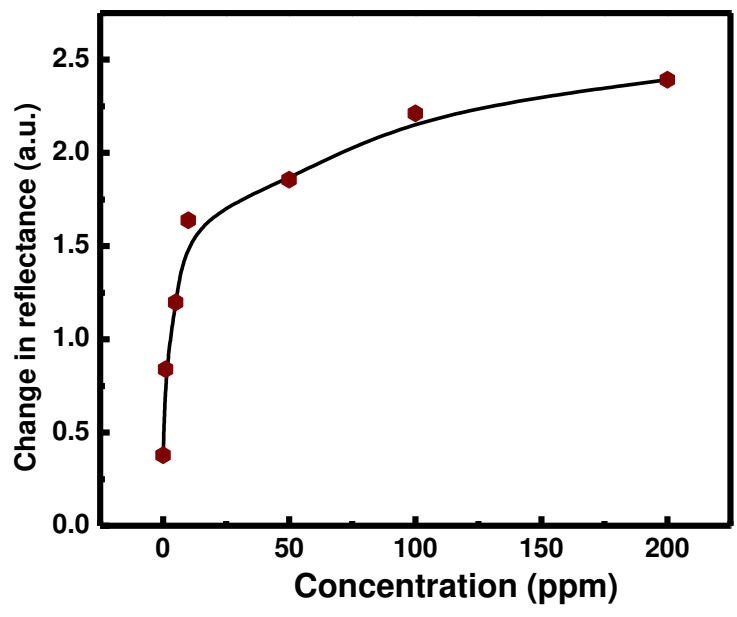

(b)

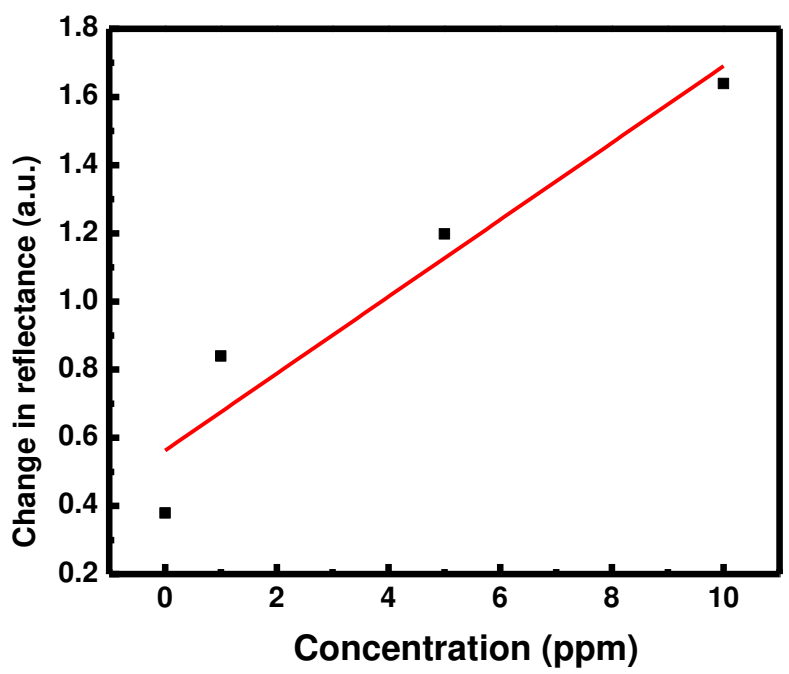

(c)

Figure 13: (a) Dynamic response of SPR gas sensor (system of prism/Au/ZnO/PPy ), (b) the calibration curve of the SPR gas sensor for the $\mathrm{NH}_{3}$ gas, and (c) linear increment of reflectance with the increasing concentration of $\mathrm{NH}_{3}$ gas

\section{Conclusion}

A specially designed gas cell has been integrated with the indigenously developed SPR set up for detection of various toxic and harmful gases. SPR system is demonstrated successfully for detection of $\mathrm{NH}_{3}$ gas using the interface of $\mathrm{ZnO}$ and $\mathrm{PPy}$. Surface plasmon resonance gas sensor system of prism/Au/ZnO/PPy, has been developed using optimized thickness of $\mathrm{ZnO}$ and PPy obtained by theoretical simulation for the detection of Ammonia $\left(\mathrm{NH}_{3}\right)$ gas. The $100 \mathrm{~nm}$ thin $\mathrm{ZnO}$ film and $150 \mathrm{~nm}$ thin PPy film were deposited which exhibits sharpest SPR reflectance curve, and is found useful for the successful detection of $\mathrm{NH}_{3}$ gas. The SPR gas sensor shows 
a fast response $(1 \mathrm{~s})$ and good sensitivity $\left(3.15 \times 10^{-3} \% / \mathrm{ppm}\right)$ towards $\mathrm{NH}_{3}$ gas over wide concentration (1 to $10 \mathrm{ppm}$ ). The dynamic response was also observed and the sensitivity was estimated from the calibration curve i.e. $0.112 / \mathrm{ppm}$. Thus, efficient SPR gas sensors are realized in the present work with enhanced response characteristics.

\section{Acknowledgements}

The Authors are highly obliged to late Prof. Vinay Gupta, Department of Physics and Astrophysics, University of Delhi for his kind help and suggestions.

\section{Funding}

Funding information is not applicable / No funding was received.

\section{Conflicts of Interest/Competing interests}

Authors declare no conflict of interest.

\section{Availability of data and material:}

The data sets generated during and/or analysed during the current study are available from the corresponding author on reasonable request.

Code Availability: Not applicable

Authors' contributions:

Ajay Pratap Singh Gahlot: Methodology, editing Conceptualization; Ayushi Paliwal: Methodology, Writing- original draft; Avinashi Kapoor: Supervision, Writing-review and editing

Ethics approval: The submitted work should be original and should not have been published elsewhere in any form or language.

Consent to participate: Not applicable

Consent for publication: Not applicable

\section{References}


1 Kato K., Dooling C. M., Shinbo K., Richardson T. H., Kaneko F., Tregonning R., Vysotsky M. O., Hunter C. A., Colloids Surf. A, 198 (2002) 811.

2 Sberveglieri G., Gas sensors- Principles, operation and developments, Kluwer Academic publisher, Dordrecht (1992).

3 Manera M. G., Rella R., Sens. Actuators B, 179 (2013) 175-186.

4 Bae M. K., Lim J. A., Kim S., Song Y. W., Opt. Exp., 21 (2013) 2018-2023.

5 Li J. P., Tredgold R. H., Jones R., Hodge P., Thin Solid films, 186 (1990) 167-176.

6 Tredgold R. H., Young M. C. J., Hodge P., Hoorfar A., IEEE Proc. I, 132 (1985) 151-156.

7 Beck U., Hertwig A., Kormunda M., Krause A., Kruger H., Lohse V., Nooke A., Pavlik J., Steinbach J., Sens. Actuators B, 160 (2011) 609-615.

8 El-Basaty A. B., El-Brolossy T. A., Abdalla S., Negm S., Abdella R. A., Talaat H., Surf. Interface Anal., 40 (2008) 1623-1626.

9 Wilde J. N., Petty M. C., Saffell J., Tempore A., Valli L., Meas. Control, 30 (1997) 269272.

10 Nikitin P. I., Beloglazov A. A., Kochergin V. E., Valeiko M. V., Ksenevich T. I., Sens. Actuators B, 54 (1999) 43-50.

11 Homola J., Yee S. S., Gauglitz G., Sens. Actuators B, 54 (1999) 3-1.

12 Yang D., Lu H. H., Chen B., Lin C. W., Sens. Actuators B, 145 (2010) 832.

13 Timmer B., Olthuis W., van den Berg A., Sens. Actuators B, 107 (2005) 666-667.

14 Cui S., Pu H., Lu G., Wen Z., Mattson E. C., Hirschmug C., Gajdardziska-Josifovska M., Weinert M., Chen J., ACS Appl. Mat. Interf., 4 (2012) 4898-4904.

15 Website: www.cdc.gov/niosh/docs

16 Maciak E., Opilski Z., Pustelny T., Mol. Quant. Acoust., 26 (2005) 205-215.

17 Dolbec R., El Khakani M. A., Sens. Lett., 3 (2005) 216-221.

18 M.K. Hossain, S.C. Ghosh, Y. Boontongkong, C. Thanachayanont, J. Dutta, "Growth of zinc oxide nanowires and nanobelts for gas sensing applications", J. of Metastable and Nanocrystalline Materials, vol. 23, pp. 27-30, 2005.

19 H. Yan, L. Zhang, J. Shen, Z. Chen, G. Shi, B. Zhang B, "Synthesis property and fieldemission behaviour of amorphous polypyrrole nanowires", Nanotechnology vol. 17, pp. 3446-3450, 2006.

20 J. H. Cho, J. B. Yu , J. S. Kim, S. O. Sohn, D. D. Lee, J. S. Huh, "Sensing behaviours of polypyrrole sensor under humidity condition", Sens Actuator B, vol. 108, pp. 389-392, 2005.

21 K. Arora, A. Chaubey, R. Singhal, R. P. Singh, M. K. Pandey, S. B. Samanta, B. D. Malhotra, S. Chand, "Application of electrochemically prepared polypyrrole-polyvinyl sulphonate films to DNA biosensor", Biosens Bioelectron, vol. 21, pp. 1777-1783, 2006.

22 A. A. Yadav, A. C. Lokhande, J. H. Kim, C. D. Lokhande, "Enhanced sensitivity and selectivity of $\mathrm{CO}_{2}$ gas sensor based on modified $\mathrm{La}_{2} \mathrm{O}_{3}$ nanorods", J Alloys Comp, vol. 723, pp. 880-886, 2017.

23 0. S. Heavens, Optical Properties of Thin Solid Films (Butterworth, London, 1955), Chap. 4.

24 A. Paliwal, A. Sharma, M. Tomar, V. Gupta, J. App. Phys. 115 (2014) 043104.

25 M.A. Chougule a, D.S. Dalavi b, Sawanta Mali b, P.S. Patil b, A.V. Moholkar b, G.L. Agawane c, J.H. Kim c, Shashwati Sen d, V.B. Patil, Measurement 45 (2012) 1989-1996. 